\title{
Orthogonal stability of functional equations with the fixed point alternative
}

\author{
Choonkil Park' , Yeol Je Cho ${ }^{2}$ and Jung Rye Lee ${ }^{3^{*}}$
}

"Correspondence: jrlee@daejin.ac.kr ${ }^{3}$ Department of Mathematics, Daejin University, Pocheon, Kyeonggi 487-711, Korea

Full list of author information is available at the end of the article

\section{Abstract}

In this paper, we investigate the orthogonal stability of functional equations in orthogonality modules over a unital Banach algebra. Using a fixed point method, we prove the Hyers-Ulam stability of the orthogonally Jensen additive functional equation

$$
2 f\left(\frac{x+y}{2}\right)=f(x)+f(y)
$$

the orthogonally Jensen quadratic functional equation

$$
2 f\left(\frac{x+y}{2}\right)+2 f\left(\frac{x-y}{2}\right)=f(x)+f(y)
$$

the orthogonally cubic functional equation

$$
f(2 x+y)+f(2 x-y)=2 f(x+y)+2 f(x-y)+12 f(x)
$$

and the orthogonally quartic functional equation

$$
f(2 x+y)+f(2 x-y)=4 f(x+y)+4 f(x-y)+24 f(x)-6 f(y)
$$

for all $x, y$ with $x \perp y$, where $\perp$ is the orthogonality in the sense of Rätz.

MSC: Primary 39B55; 47H10; 39B52; 46H25

Keywords: Hyers-Ulam stability; orthogonally (Jensen additive, Jensen quadratic, cubic, quartic) functional equation; fixed point; orthogonality module over Banach algebra; orthogonality space

\section{Introduction and preliminaries}

Assume that $X$ is a real inner product space and $f: X \rightarrow \mathbb{R}$ is a solution of the orthogonal Cauchy functional equation $f(x+y)=f(x)+f(y)$, where $\langle x, y\rangle=0$. By the Pythagorean theorem, $f(x)=\|x\|^{2}$ is a solution of the conditional equation. Of course, this function does not satisfy the additivity equation everywhere. Thus the orthogonal Cauchy equation is not equivalent to the classic Cauchy equation on the whole inner product space.

Pinsker [1] characterized orthogonally additive functionals on an inner product space when the orthogonality is the ordinary one in such spaces. Sundaresan [2] generalized this result to arbitrary Banach spaces equipped with the Birkhoff-James orthogonality. 
The orthogonal Cauchy functional equation

$$
f(x+y)=f(x)+f(y), \quad x \perp y,
$$

in which $\perp$ is an abstract orthogonality relation, was first investigated by Gudder and Strawther [3]. They defined $\perp$ by a system consisting of five axioms and described the general semi-continuous real-valued solution of the conditional Cauchy functional equation. In 1985, Rätz [4] introduced a new definition of orthogonality by using more restrictive axioms than Gudder and Strawther. Moreover, he investigated the structure of orthogonally additive mappings. Rätz and Szabó [5] investigated the problem in a rather more general framework.

Let us recall the orthogonality in the sense of Rätz [4].

Suppose that $X$ is a real vector space (algebraic module) with $\operatorname{dim} X \geq 2$, and $\perp$ is a binary relation on $X$ with the following properties:

$\left(O_{1}\right)$ Totality of $\perp$ for zero: $x \perp 0$ and $0 \perp x$ for all $x \in X$;

$\left(O_{2}\right)$ Independence: if $x, y \in X-\{0\}$ and $x \perp y$, then $x$ and $y$ are linearly independent;

$\left(O_{3}\right)$ Homogeneity: if $x, y \in X$ and $x \perp y$, then $\alpha x \perp \beta y$ for all $\alpha, \beta \in \mathbb{R}$;

$\left(\mathrm{O}_{4}\right)$ Thalesian property: if $P$ is a 2-dimensional subspace of $X, x \in P$ and $\lambda \in \mathbb{R}_{+}$, which is the set of nonnegative real numbers, then there exists $y_{0} \in P$ such that $x \perp y_{0}$ and $x+y_{0} \perp \lambda x-y_{0}$.

The pair $(X, \perp)$ is called an orthogonality space (resp., module). By an orthogonality normed space (normed module) we mean an orthogonality space (resp., module) having a normed (resp., normed module) structure.

Some interesting examples are as follows:

(1) The trivial orthogonality on a vector space $X$ defined by $\left(O_{1}\right)$ and, for any non-zero elements $x, y \in X, x \perp y$ if and only if $x, y$ are linearly independent.

(2) The ordinary orthogonality on an inner product space $(X,\langle\cdot, \cdot\rangle)$ given by $x \perp y$ if and only if $\langle x, y\rangle=0$.

(3) The Birkhoff-James orthogonality on a normed space $(X,\|\cdot\|)$ defined by $x \perp y$ if and only if $\|x+\lambda y\| \geq\|x\|$ for all $\lambda \in \mathbb{R}$.

The relation $\perp$ is called symmetric if $x \perp y$ implies that $y \perp x$ for all $x, y \in X$. Clearly, Examples (1) and (2) are symmetric, but Example (3) is not. It is remarkable to note, however, that a real normed space of a dimension greater than 2 is an inner product space if and only if the Birkhoff-James orthogonality is symmetric. There are several orthogonality notions on a real normed space such as Birkhoff-James, Boussouis, Singer, Carlsson, unitary-Boussouis, Roberts, Phythagorean, isosceles and Diminnie (see [6-12]).

The stability problem of functional equations originated from the following question of Ulam [13]: Under what condition is there an additive mapping near an approximately additive mapping? In 1941, Hyers [14] gave a partial affirmative answer to the question of Ulam in the context of Banach spaces. In 1978, Rassias [15] extended the theorem of Hyers by considering the unbounded Cauchy difference

$$
\|f(x+y)-f(x)-f(y)\| \leq \varepsilon\left(\|x\|^{p}+\|y\|^{p}\right) \quad(\varepsilon>0, p \in[0,1)) .
$$


During the last decades, several stability problems of functional equations have been investigated in the spirit of Hyers-Ulam-Rassias. The readers refer to [16-20] and references therein for detailed information on the stability of functional equations.

Ger and Sikorska [21] investigated the orthogonal stability of the Cauchy functional equation $f(x+y)=f(x)+f(y)$, namely they showed that, if $f$ is a mapping from an orthogonality space $X$ into a real Banach space $Y$ and

$$
\|f(x+y)-f(x)-f(y)\| \leq \varepsilon
$$

for all $x, y \in X$ with $x \perp y$ and for some $\varepsilon>0$, then there exists exactly one orthogonally additive mapping $g: X \rightarrow Y$ such that $\|f(x)-g(x)\| \leq \frac{16}{3} \varepsilon$ for all $x \in X$.

The first author treating the stability of the quadratic equation was Skof [22] by proving that, if $f$ is a mapping from a normed space $X$ into a Banach space $Y$ satisfying

$$
\|f(x+y)+f(x-y)-2 f(x)-2 f(y)\| \leq \varepsilon
$$

for some $\varepsilon>0$, then there is a unique quadratic mapping $g: X \rightarrow Y$ such that $\| f(x)-$ $g(x) \| \leq \frac{\varepsilon}{2}$. Cholewa [23] extended Skof's theorem by replacing $X$ by an abelian group $G$. Skof's result was later generalized by Czerwik [24] in the spirit of Hyers-Ulam-Rassias. The stability problem of functional equations has been extensively investigated by some mathematicians (see [25-31]).

The orthogonally quadratic equation

$$
f(x+y)+f(x-y)=2 f(x)+2 f(y), \quad x \perp y
$$

was first investigated by Vajzović [32] when $X$ is a Hilbert space, $Y$ is the scalar field, $f$ is continuous and $\perp$ means the Hilbert space orthogonality. Later, Drljević [33], Fochi [34], Moslehian [35, 36] and Szabó [37] generalized this result (see also [38-40]).

In [41], Jun and Kim considered the following cubic functional equation:

$$
f(2 x+y)+f(2 x-y)=2 f(x+y)+2 f(x-y)+12 f(x) .
$$

It is easy to show that the function $f(x)=x^{3}$ satisfies the functional equation (1.1), which is called a cubic functional equation, and every solution of the cubic functional equation is said to be a cubic mapping.

Let $X$ be an orthogonality space and $Y$ be a real Banach space. A mapping $f: X \rightarrow Y$ is called orthogonally cubic if it satisfies the orthogonally cubic functional equation

$$
f(2 x+y)+f(2 x-y)=2 f(x+y)+2 f(x-y)+12 f(x)
$$

for all $x, y$ with $x \perp y$.

In [42], Lee et al. considered the following quartic functional equation:

$$
f(2 x+y)+f(2 x-y)=4 f(x+y)+4 f(x-y)+24 f(x)-6 f(y) .
$$


It is easy to show that the function $f(x)=x^{4}$ satisfies the functional equation (1.3), which is called a quartic functional equation, and every solution of the quartic functional equation is said to be a quartic mapping (for the stability of the ACQ and quartic functional equations, see $[26,31]$ and others).

Let $X$ be an orthogonality space and $Y$ be a Banach space. A mapping $f: X \rightarrow Y$ is called orthogonally quartic if it satisfies the orthogonally quartic functional equation

$$
f(2 x+y)+f(2 x-y)=4 f(x+y)+4 f(x-y)+24 f(x)-6 f(y)
$$

for all $x, y$ with $x \perp y$.

Let $X$ be a set. A function $d: X \times X \rightarrow[0, \infty]$ is called a generalized metric on $X$ if $d$ satisfies the following conditions:

(1) $d(x, y)=0$ if and only if $x=y$;

(2) $d(x, y)=d(y, x)$ for all $x, y \in X$;

(3) $d(x, z) \leq d(x, y)+d(y, z)$ for all $x, y, z \in X$.

We recall a fundamental result in a fixed point theory.

Theorem $1.1([43,44])$ Let $(X, d)$ be a complete generalized metric space and $J: X \rightarrow X$ be a strictly contractive mapping with the Lipschitz constant $\alpha<1$. Then, for each given element $x \in X$, either

$$
d\left(J^{n} x, J^{n+1} x\right)=\infty
$$

for all nonnegative integers $n$ or there exists a positive integer $n_{0}$ such that

(1) $d\left(J^{n} x, J^{n+1} x\right)<\infty$ for all $n \geq n_{0}$;

(2) the sequence $\left\{J^{n} x\right\}$ converges to a fixed point $y^{\prime \prime}$ of $J$;

(3) $y^{*}$ is the unique fixed point of $J$ in the set $Y=\left\{y \in X \mid d\left(J^{n_{0}} x, y\right)<\infty\right\}$;

(4) $d\left(y, y^{*}\right) \leq \frac{1}{1-\alpha} d(y, J y)$ for all $y \in Y$.

In 1996, Isac and Rassias [45] were the first to provide applications of the stability theory of functional equations for the proof of new fixed point theorems with applications. By using fixed point methods, the stability problems of several functional equations have been extensively investigated by a number of authors (see [46-55]).

This paper is organized as follows. In Section 2, we prove the Hyers-Ulam stability of the orthogonally Jensen additive functional equation in orthogonality modules over a unital Banach algebra. In Section 3, we prove the Hyers-Ulam stability of the orthogonally Jensen quadratic functional equation in orthogonality modules over a unital Banach algebra. In Section 4, we prove the Hyers-Ulam stability of the orthogonally cubic functional equation (1.2) in orthogonality modules over a unital Banach algebra. In Section 5, we prove the Hyers-Ulam stability of the orthogonally quartic functional equation (1.4) in orthogonality modules over a unital Banach algebra.

Throughout this paper, assume that $(X, \perp)$ is an orthogonality module over a unital Banach algebra $A$ and that $\left(Y,\|\cdot\|_{Y}\right)$ is a real Banach module over $A$. Let $A_{1}:=\{u \in A \mid$ $\|u\|=1\}$, and $e$ be the unity of $A$. 


\section{Stability of the orthogonally Jensen additive functional equation}

In this section, applying some ideas from $[18,21]$, we deal with the stability problem for the orthogonally Jensen additive functional equation

$$
2 f\left(\frac{x+y}{2}\right)=f(x)+f(y)
$$

for all $x, y \in X$ with $x \perp y$.

Definition 2.1 An additive mapping $f: X \rightarrow Y$ is called an $A$-additive mapping if $f(a x)=$ $a f(x)$ for all $a \in A$ and $x \in X$.

Theorem 2.2 Let $\varphi: X^{2} \rightarrow[0, \infty)$ be a function such that there exists an $\alpha<1$ with

$$
\varphi(x, y) \leq 2 \alpha \varphi\left(\frac{x}{2}, \frac{y}{2}\right)
$$

for all $x, y \in X$ with $x \perp y$. Let $f: X \rightarrow Y$ be a mapping satisfying $f(0)=0$ and

$$
\left\|2 a f\left(\frac{x+y}{2}\right)-f(a x)-f(a y)\right\|_{Y} \leq \varphi(x, y)
$$

for all $a \in A_{1}$ and all $x, y \in X$ with $x \perp y$. If, for each $x \in X$, the mapping $f(t x)$ is continuous in $t \in \mathbb{R}$, then there exists a unique orthogonally Jensen $A$-additive mapping $L: X \rightarrow Y$ such that

$$
\|f(x)-L(x)\|_{Y} \leq \frac{\alpha}{1-\alpha} \varphi(x, 0)
$$

for all $x \in X$.

Proof Putting $y=0$ and $a=e$ in (2.2), we get

$$
\left\|2 f\left(\frac{x}{2}\right)-f(x)\right\|_{Y} \leq \varphi(x, 0)
$$

for all $x \in X$ since $x \perp 0$. So, we have

$$
\left\|f(x)-\frac{1}{2} f(2 x)\right\|_{Y} \leq \frac{1}{2} \varphi(2 x, 0) \leq \alpha \cdot \varphi(x, 0)
$$

for all $x \in X$. Consider the set

$$
S:=\{h: X \rightarrow Y\}
$$

and introduce the generalized metric on $S$ :

$$
d(g, h)=\inf \left\{\mu \in \mathbb{R}_{+}:\|g(x)-h(x)\|_{Y} \leq \mu \varphi(x, 0), \forall x \in X\right\},
$$

where, as usual, $\inf \phi=+\infty$. It is easy to show that $(S, d)$ is complete (see [50]). 
Now, we consider the linear mapping $J: S \rightarrow S$ such that

$$
J g(x):=\frac{1}{2} g(2 x)
$$

for all $x \in X$. Let $g, h \in S$ be given such that $d(g, h)=\varepsilon$. Then we have

$$
\|g(x)-h(x)\|_{Y} \leq \varphi(x, 0)
$$

for all $x \in X$. Hence,

$$
\|J g(x)-J h(x)\|_{Y}=\left\|\frac{1}{2} g(2 x)-\frac{1}{2} h(2 x)\right\|_{Y} \leq \alpha \varphi(x, 0)
$$

for all $x \in X$. So, $d(g, h)=\varepsilon$ implies that $d(J g, J h) \leq \alpha \varepsilon$. This means that

$$
d(J g, J h) \leq \alpha d(g, h)
$$

for all $g, h \in S$. It follows from (2.5) that $d(f, J f) \leq \alpha$. By Theorem 1.1, there exists a mapping $L: X \rightarrow Y$ satisfying the following:

(1) $L$ is a fixed point of $J$, i.e.,

$$
L(2 x)=2 L(x)
$$

for all $x \in X$. The mapping $L$ is a unique fixed point of $J$ in the set

$$
M=\{g \in S: d(h, g)<\infty\}
$$

This implies that $L$ is a unique mapping satisfying (2.6) such that there exists a $\mu \in(0, \infty)$ satisfying

$$
\|f(x)-L(x)\|_{Y} \leq \mu \varphi(x, 0)
$$

for all $x \in X$;

(2) $d\left(J^{n} f, L\right) \rightarrow 0$ as $n \rightarrow \infty$. This implies the equality

$$
\lim _{n \rightarrow \infty} \frac{1}{2^{n}} f\left(2^{n} x\right)=L(x)
$$

for all $x \in X$;

(3) $d(f, L) \leq \frac{1}{1-\alpha} d(f, J f)$, which implies the inequality

$$
d(f, L) \leq \frac{\alpha}{1-\alpha}
$$

This implies that inequality (2.3) holds. 
Let $a=e$ in (2.2). It follows from (2.1) and (2.2) that

$$
\begin{aligned}
\left\|2 L\left(\frac{x+y}{2}\right)-L(x)-L(y)\right\|_{Y} & =\lim _{n \rightarrow \infty} \frac{1}{2^{n}}\left\|2 f\left(2^{n-1}(x+y)\right)-f\left(2^{n} x\right)-f\left(2^{n} y\right)\right\|_{Y} \\
& \leq \lim _{n \rightarrow \infty} \frac{1}{2^{n}} \varphi\left(2^{n} x, 2^{n} y\right) \leq \lim _{n \rightarrow \infty} \frac{2^{n} \alpha^{n}}{2^{n}} \varphi(x, y)=0
\end{aligned}
$$

for all $x, y \in X$ with $x \perp y$. So,

$$
2 L\left(\frac{x+y}{2}\right)-L(x)-L(y)=0
$$

for all $x, y \in X$ with $x \perp y$. Hence, $L: X \rightarrow Y$ is an orthogonally Jensen additive mapping. Let $y=0$ in (2.2). It follows from (2.1) and (2.2) that

$$
\begin{aligned}
\left\|2 a L\left(\frac{x}{2}\right)-L(a x)\right\|_{Y} & =\lim _{n \rightarrow \infty} \frac{1}{2^{n}}\left\|2 a f\left(2^{n-1} x\right)-f\left(2^{n} a x\right)\right\|_{Y} \\
& \leq \lim _{n \rightarrow \infty} \frac{1}{2^{n}} \varphi\left(2^{n} x, 0\right) \leq \lim _{n \rightarrow \infty} \frac{2^{n} \alpha^{n}}{2^{n}} \varphi(x, 0)=0
\end{aligned}
$$

for all $x \in X$. So, we have

$$
2 a L\left(\frac{x}{2}\right)-L(a x)=0
$$

for all $x \in X$, and hence

$$
L(a x)=2 a L\left(\frac{x}{2}\right)=a L(x)
$$

for all $a \in A_{1}$ and $x \in X$. By the same reasoning as in the proof of Theorem in [15], we can show that $L: X \rightarrow Y$ is $\mathbb{R}$-linear since the mapping $f(t x)$ is continuous in $t \in \mathbb{R}$ for each $x \in X$. For each $a \in A$ with $a \neq 0$, we have

$$
L(a x)=L\left(\|a\| \frac{a}{\|a\|} x\right)=\|a\| L\left(\frac{a}{\|a\|} x\right)=\|a\| \frac{a}{\|a\|} L(x)=a L(x)
$$

for all $x \in X$. Thus $L: X \rightarrow Y$ is a unique orthogonally Jensen $A$-additive mapping satisfying (2.3). This completes the proof.

From now on, in corollaries, assume that $(X, \perp)$ is an orthogonality normed module over a unital Banach algebra $A$.

Corollary 2.3 Let $\theta$ be a positive real number and $p$ be a real number with $0<p<1$. Let $f: X \rightarrow Y$ be a mapping satisfying $f(0)=0$ and

$$
\left\|2 a f\left(\frac{x+y}{2}\right)-f(a x)-f(a y)\right\|_{Y} \leq \theta\left(\|x\|^{p}+\|y\|^{p}\right)
$$

for all $a \in A_{1}$ and $x, y \in X$ with $x \perp y$. If, for each $x \in X$, the mapping $f(t x)$ is continuous in $t \in \mathbb{R}$, then there exists a unique orthogonally Jensen A-additive mapping $L: X \rightarrow Y$ such 
that

$$
\|f(x)-L(x)\|_{Y} \leq \frac{2^{p} \theta}{2-2^{p}}\|x\|^{p}
$$

for all $x \in X$.

Proof The proof follows from Theorem 2.2 by taking $\varphi(x, y)=\theta\left(\|x\|^{p}+\|y\|^{p}\right)$ for all $x, y \in X$ with $x \perp y$. Then we can choose $\alpha=2^{p-1}$ and we get the desired result.

Theorem 2.4 Let $f: X \rightarrow Y$ be a mapping satisfying (2.2) and $f(0)=0$ for which there exists a function $\varphi: X^{2} \rightarrow[0, \infty)$ such that

$$
\varphi(x, y) \leq \frac{\alpha}{2} \varphi(2 x, 2 y)
$$

for all $x, y \in X$ with $x \perp y$. If, for each $x \in X$, the mapping $f(t x)$ is continuous in $t \in \mathbb{R}$, then there exists a unique orthogonally Jensen A-additive mapping $L: X \rightarrow Y$ such that

$$
\|f(x)-L(x)\|_{Y} \leq \frac{1}{1-\alpha} \varphi(x, 0)
$$

for all $x \in X$.

Proof Let $(S, d)$ be the generalized metric space defined in the proof of Theorem 2.2.

Now, we consider the linear mapping $J: S \rightarrow S$ such that

$$
J g(x):=2 g\left(\frac{x}{2}\right)
$$

for all $x \in X$. It follows from $(2.4)$ that $d(f, J f) \leq 1$. So,

$$
d(f, L) \leq \frac{1}{1-\alpha}
$$

Thus we obtain inequality (2.8). The rest of the proof is similar to the proof of Theorem 2.2. This completes the proof.

Corollary 2.5 Let $\theta$ be a positive real number and $p$ be a real number with $p>1$. Let $f: X \rightarrow Y$ be a mapping satisfying $f(0)=0$ and (2.7). If, for each $x \in X$, the mapping $f(t x)$ is continuous in $t \in \mathbb{R}$, then there exists a unique orthogonally Jensen A-additive mapping $L: X \rightarrow Y$ such that

$$
\|f(x)-L(x)\|_{Y} \leq \frac{2^{p} \theta}{2^{p}-2}\|x\|^{p}
$$

for all $x \in X$.

Proof The proof follows from Theorem 2.4 by taking $\varphi(x, y)=\theta\left(\|x\|^{p}+\|y\|^{p}\right)$ for all $x, y \in X$ with $x \perp y$. Then we can choose $\alpha=2^{1-p}$ and we get the desired result. 


\section{Stability of the orthogonally Jensen quadratic functional equation}

In this section, applying some ideas from $[18,21]$, we deal with the stability problem for the orthogonally Jensen quadratic functional equation

$$
2 f\left(\frac{x+y}{2}\right)+2 f\left(\frac{x-y}{2}\right)=f(x)+f(y)
$$

for all $x, y \in X$ with $x \perp y$.

Definition 3.1 A quadratic mapping $f: X \rightarrow Y$ is called an A-quadratic mapping if $f(a x)=a^{2} f(x)$ for all $a \in A$ and $x \in X$.

Theorem 3.2 Let $\varphi: X^{2} \rightarrow[0, \infty)$ be a function such that there exists an $\alpha<1$ with

$$
\varphi(x, y) \leq 4 \alpha \varphi\left(\frac{x}{2}, \frac{y}{2}\right)
$$

for all $x, y \in X$ with $x \perp y$. Let $f: X \rightarrow Y$ be a mapping satisfying $f(0)=0$ and

$$
\left\|2 a^{2} f\left(\frac{x+y}{2}\right)+2 a^{2} f\left(\frac{x-y}{2}\right)-f(a x)-f(a y)\right\|_{Y} \leq \varphi(x, y)
$$

for all $a \in A_{1}$ and $x, y \in X$ with $x \perp y$. If, for each $x \in X$, the mapping $f(t x)$ is continuous in $t \in \mathbb{R}$, then there exists a unique orthogonally Jensen A-quadratic mapping $Q: X \rightarrow Y$ such that

$$
\|f(x)-Q(x)\|_{Y} \leq \frac{\alpha}{1-\alpha} \varphi(x, 0)
$$

for all $x \in X$.

Proof Putting $y=0$ and $a=e$ in (3.2), we get

$$
\left\|4 f\left(\frac{x}{2}\right)-f(x)\right\|_{Y} \leq \varphi(x, 0)
$$

for all $x \in X$, since $x \perp 0$. So, we have

$$
\left\|f(x)-\frac{1}{4} f(2 x)\right\|_{Y} \leq \frac{1}{4} \varphi(2 x, 0) \leq \alpha \cdot \varphi(x, 0)
$$

for all $x \in X$. By the same reasoning as in the proof of Theorem 2.2, one can obtain an orthogonally Jensen quadratic mapping $Q: X \rightarrow Y$ defined by

$$
\lim _{n \rightarrow \infty} \frac{1}{4^{n}} f\left(2^{n} x\right)=Q(x)
$$

for all $x \in X$.

Let $(S, d)$ be the generalized metric space defined in the proof of Theorem 2.2.

Now, we consider the linear mapping $J: S \rightarrow S$ such that

$$
J g(x):=\frac{1}{4} g(2 x)
$$


for all $x \in X$. It follows from (3.5) that $d(f, J f) \leq \alpha$. So,

$$
d(f, Q) \leq \frac{\alpha}{1-\alpha} .
$$

Thus we obtain inequality (3.3). Let $y=0$ in (3.2). It follows from (3.1) and (3.2) that

$$
\begin{aligned}
\left\|4 a^{2} Q\left(\frac{x}{2}\right)-Q(a x)\right\|_{Y} & =\lim _{n \rightarrow \infty} \frac{1}{4^{n}}\left\|4 a^{2} f\left(2^{n-1} x\right)-f\left(2^{n} a x\right)\right\|_{Y} \\
& \leq \lim _{n \rightarrow \infty} \frac{1}{4^{n}} \varphi\left(2^{n} x, 0\right) \leq \frac{4^{n} \alpha^{n}}{4^{n}} \varphi(x, 0)=0
\end{aligned}
$$

for all $x \in X$. So, we have

$$
4 a^{2} Q\left(\frac{x}{2}\right)-Q(a x)=0
$$

for all $x \in X$, and hence

$$
Q(a x)=4 a^{2} Q\left(\frac{x}{2}\right)=a^{2} Q(x)
$$

for all $a \in A_{1}$ and $x \in X$. By the same reasoning as in the proof of [15, Theorem], we can show that, for each $t \in \mathbb{R}, Q: X \rightarrow Y$ satisfies $Q(t x)=t^{2} Q(x)$ all $x \in X$ since the mapping $f(t x)$ is continuous in $t \in \mathbb{R}$ for each $x \in X$. For each $a \in A$ with $a \neq 0$, we have

$$
Q(a x)=Q\left(\|a\| \frac{a}{\|a\|} x\right)=\|a\|^{2} Q\left(\frac{a}{\|a\|} x\right)=\|a\|^{2} \frac{a^{2}}{\|a\|^{2}} Q(x)=a^{2} Q(x)
$$

for all $x \in X$. Thus $Q: X \rightarrow Y$ is a unique orthogonally Jensen $A$-quadratic mapping satisfying (3.3). This completes the proof.

Corollary 3.3 Let $\theta$ be a positive real number and $p$ be a real number with $0<p<2$. Let $f: X \rightarrow Y$ be a mapping satisfying

$$
\left\|2 a^{2} f\left(\frac{x+y}{2}\right)+2 a^{2} f\left(\frac{x-y}{2}\right)-f(a x)-f(a y)\right\|_{Y} \leq \theta\left(\|x\|^{p}+\|y\|^{p}\right)
$$

for all $a \in A_{1}$ and $x, y \in X$ with $x \perp y$. If, for each $x \in X$, the mapping $f(t x)$ is continuous in $t \in \mathbb{R}$, then there exists a unique orthogonally Jensen A-quadratic mapping $Q: X \rightarrow Y$ such that

$$
\|f(x)-Q(x)\|_{Y} \leq \frac{2^{p} \theta}{4-2^{p}}\|x\|^{p}
$$

for all $x \in X$.

Proof The proof follows from Theorem 3.2 by taking $\varphi(x, y)=\theta\left(\|x\|^{p}+\|y\|^{p}\right)$ for all $x, y \in X$ with $x \perp y$. Then we can choose $\alpha=2^{p-2}$ and we get the desired result. 
Theorem 3.4 Let $f: X \rightarrow Y$ be a mapping satisfying (3.2) and $f(0)=0$ for which there exists a function $\varphi: X^{2} \rightarrow[0, \infty)$ such that

$$
\varphi(x, y) \leq \frac{\alpha}{4} \varphi(2 x, 2 y)
$$

for all $x, y \in X$ with $x \perp y$. If, for each $x \in X$, the mapping $f(t x)$ is continuous in $t \in \mathbb{R}$, then there exists a unique orthogonally Jensen A-quadratic mapping $Q: X \rightarrow Y$ such that

$$
\|f(x)-Q(x)\|_{Y} \leq \frac{1}{1-\alpha} \varphi(x, 0)
$$

for all $x \in X$.

Proof Let $(S, d)$ be the generalized metric space defined in the proof of Theorem 2.2.

Now, we consider the linear mapping $J: S \rightarrow S$ such that

$$
J g(x):=4 g\left(\frac{x}{2}\right)
$$

for all $x \in X$. It follows from (3.4) that $d(f, J f) \leq 1$. So, we obtain inequality (3.7). The rest of the proof is similar to the proofs of Theorems 2.2 and 3.2.

Corollary 3.5 Let $\theta$ be a positive real number and $p$ be a real number with $p>2$. Let $f: X \rightarrow Y$ be a mapping satisfying (3.6). If, for each $x \in X$, the mapping $f(t x)$ is continuous in $t \in \mathbb{R}$, then there exists a unique orthogonally Jensen A-quadratic mapping $Q: X \rightarrow Y$ such that

$$
\|f(x)-Q(x)\|_{Y} \leq \frac{2^{p} \theta}{2^{p}-4}\|x\|^{p}
$$

for all $x \in X$.

Proof The proof follows from Theorem 3.4 by taking $\varphi(x, y)=\theta\left(\|x\|^{p}+\|y\|^{p}\right)$ for all $x, y \in X$ with $x \perp y$. Then we can choose $\alpha=2^{2-p}$ and we get the desired result.

\section{Stability of the orthogonally cubic functional equation}

In this section, applying some ideas from $[18,21]$, we deal with the stability problem for the orthogonally cubic functional equation

$$
f(2 x+y)+f(2 x-y)=2 f(x+y)+2 f(x-y)+12 f(x)
$$

for all $x, y \in X$ with $x \perp y$.

Definition 4.1 A cubic mapping $f: X \rightarrow Y$ is called an $A$-cubic mapping if $f(a x)=a^{3} f(x)$ for all $a \in A$ and $x \in X$.

Theorem 4.2 Let $\varphi: X^{2} \rightarrow[0, \infty)$ be a function such that there exists an $\alpha<1$ with

$$
\varphi(x, y) \leq 8 \alpha \varphi\left(\frac{x}{2}, \frac{y}{2}\right)
$$


for all $x, y \in X$ with $x \perp y$. Let $f: X \rightarrow Y$ be a mapping satisfying $f(0)=0$ and

$$
\begin{aligned}
& \left\|a^{3} f(2 x+y)+a^{3} f(2 x-y)-2 f(a x+a y)-2 f(a x-a y)-12 f(a x)\right\|_{Y} \\
& \quad \leq \varphi(x, y)
\end{aligned}
$$

for all $a \in A_{1}$ and $x, y \in X$ with $x \perp y$. If, for each $x \in X$, the mapping $f(t x)$ is continuous in $t \in \mathbb{R}$, then there exists a unique orthogonally $A$-cubic mapping $C: X \rightarrow Y$ such that

$$
\|f(x)-C(x)\|_{Y} \leq \frac{1}{16-16 \alpha} \varphi(x, 0)
$$

for all $x \in X$.

Proof Putting $y=0$ and $a=e$ in (4.1), we get

$$
\|2 f(2 x)-16 f(x)\|_{Y} \leq \varphi(x, 0)
$$

for all $x \in X$ since $x \perp 0$. So, we have

$$
\left\|f(x)-\frac{1}{8} f(2 x)\right\|_{Y} \leq \frac{1}{16} \varphi(x, 0)
$$

for all $x \in X$.

Let $(S, d)$ be the generalized metric space defined in the proof of Theorem 2.2.

Now, we consider the linear mapping $J: S \rightarrow S$ such that

$$
J g(x):=\frac{1}{8} g(2 x)
$$

for all $x \in X$. The rest of the proof is similar to the proofs of Theorems 2.2 and 3.2. This completes the proof.

Corollary 4.3 Let $\theta$ be a positive real number and $p$ be a real number with $0<p<3$. Let $f: X \rightarrow Y$ be a mapping satisfying

$$
\begin{aligned}
& \left\|a^{3} f(2 x+y)+a^{3} f(2 x-y)-2 f(a x+a y)-2 f(a x-a y)-12 f(a x)\right\|_{Y} \\
& \quad \leq \theta\left(\|x\|^{p}+\|y\|^{p}\right)
\end{aligned}
$$

for all $a \in A_{1}$ and $x, y \in X$ with $x \perp y$. If, for each $x \in X$, the mapping $f(t x)$ is continuous in $t \in \mathbb{R}$, then there exists a unique orthogonally $A$-cubic mapping $C: X \rightarrow Y$ such that

$$
\|f(x)-C(x)\|_{Y} \leq \frac{\theta}{2\left(8-2^{p}\right)}\|x\|^{p}
$$

for all $x \in X$.

Proof The proof follows from Theorem 4.2 by taking $\varphi(x, y)=\theta\left(\|x\|^{p}+\|y\|^{p}\right)$ for all $x, y \in X$ with $x \perp y$. Then we can choose $\alpha=2^{p-3}$ and we get the desired result. 
Theorem 4.4 Let $f: X \rightarrow Y$ be a mapping satisfying (4.1) and $f(0)=0$ for which there exists a function $\varphi: X^{2} \rightarrow[0, \infty)$ such that

$$
\varphi(x, y) \leq \frac{\alpha}{8} \varphi(2 x, 2 y)
$$

for all $x, y \in X$ with $x \perp y$. If, for each $x \in X$, the mapping $f(t x)$ is continuous in $t \in \mathbb{R}$, then there exists a unique orthogonally A-cubic mapping $C: X \rightarrow Y$ such that

$$
\|f(x)-C(x)\|_{Y} \leq \frac{\alpha}{16-16 \alpha} \varphi(x, 0)
$$

for all $x \in X$.

Proof Let $(S, d)$ be the generalized metric space defined in the proof of Theorem 2.2.

Now, we consider the linear mapping $J: S \rightarrow S$ such that

$$
J g(x):=8 g\left(\frac{x}{2}\right)
$$

for all $x \in X$. It follows from (4.2) that $d(f, J f) \leq \frac{\alpha}{16}$. So, we obtain inequality (4.4). The rest of the proof is similar to the proofs of Theorems 2.2 and 3.2.

Corollary 4.5 Let $\theta$ be a positive real number and $p$ be a real number with $p>3$. Let $f: X \rightarrow Y$ be a mapping satisfying (4.3). If, for each $x \in X$, the mapping $f(t x)$ is continuous in $t \in \mathbb{R}$, then there exists a unique orthogonally A-cubic mapping $C: X \rightarrow Y$ such that

$$
\|f(x)-C(x)\|_{Y} \leq \frac{\theta}{2\left(2^{p}-8\right)}\|x\|^{p}
$$

for all $x \in X$.

Proof The proof follows from Theorem 4.4 by taking $\varphi(x, y)=\theta\left(\|x\|^{p}+\|y\|^{p}\right)$ for all $x, y \in X$ with $x \perp y$. Then we can choose $\alpha=2^{3-p}$ and we get the desired result.

\section{Stability of the orthogonally quartic functional equation}

Applying some ideas from $[18,21]$, we deal with the stability problem for the orthogonally quartic functional equation

$$
f(2 x+y)+f(2 x-y)=4 f(x+y)+4 f(x-y)+24 f(x)-6 f(y)
$$

for all $x, y \in X$ with $x \perp y$.

Definition 5.1 A quartic mapping $f: X \rightarrow Y$ is called an A-quartic mapping if $f(a x)=$ $a^{4} f(x)$ for all $a \in A$ and $x \in X$.

Theorem 5.2 Let $\varphi: X^{2} \rightarrow[0, \infty)$ be a function such that there exists an $\alpha<1$ with

$$
\varphi(x, y) \leq 16 \alpha \varphi\left(\frac{x}{2}, \frac{y}{2}\right)
$$


for all $x, y \in X$ with $x \perp y$. Let $f: X \rightarrow Y$ be a mapping satisfying $f(0)=0$ and

$$
\begin{aligned}
& \left\|a^{4} f(2 x+y)+a^{4} f(2 x-y)-4 f(a x+a y)-4 f(a x-a y)-24 f(a x)+6 f(a y)\right\|_{Y} \\
& \quad \leq \varphi(x, y)
\end{aligned}
$$

for all $a \in A_{1}$ and $x, y \in X$ with $x \perp y$. If, for each $x \in X$, the mapping $f(t x)$ is continuous in $t \in \mathbb{R}$, then there exists a unique orthogonally A-quartic mapping $P: X \rightarrow Y$ such that

$$
\|f(x)-P(x)\|_{Y} \leq \frac{1}{32-32 \alpha} \varphi(x, 0)
$$

for all $x \in X$.

Proof Putting $y=0$ and $a=e$ in (5.1), we get

$$
\|2 f(2 x)-32 f(x)\|_{Y} \leq \varphi(x, 0)
$$

for all $x \in X$, since $x \perp 0$. So, we have

$$
\left\|f(x)-\frac{1}{16} f(2 x)\right\|_{Y} \leq \frac{1}{32} \varphi(x, 0)
$$

for all $x \in X$.

Let $(S, d)$ be the generalized metric space defined in the proof of Theorem 2.2.

Now, we consider the linear mapping $J: S \rightarrow S$ such that

$$
J g(x):=\frac{1}{16} g(2 x)
$$

for all $x \in X$. The rest of the proof is similar to the proofs of Theorems 2.2 and 3.2.

Corollary 5.3 Let $\theta$ be a positive real number and $p$ be a real number with $0<p<4$. Let $f: X \rightarrow Y$ be a mapping satisfying

$$
\begin{aligned}
& \left\|a^{4} f(2 x+y)+a^{4} f(2 x-y)-4 f(a x+a y)-4 f(a x-a y)-24 f(a x)+6 f(a y)\right\|_{Y} \\
& \quad \leq \theta\left(\|x\|^{p}+\|y\|^{p}\right)
\end{aligned}
$$

for all $a \in A_{1}$ and $x, y \in X$ with $x \perp y$. If, for each $x \in X$, the mapping $f(t x)$ is continuous in $t \in \mathbb{R}$, then there exists a unique orthogonally $A$-quartic mapping $P: X \rightarrow Y$ such that

$$
\|f(x)-P(x)\|_{Y} \leq \frac{\theta}{2\left(16-2^{p}\right)}\|x\|^{p}
$$

for all $x \in X$.

Proof The proof follows from Theorem 5.2 by taking $\varphi(x, y)=\theta\left(\|x\|^{p}+\|y\|^{p}\right)$ for all $x, y \in X$ with $x \perp y$. Then we can choose $\alpha=2^{p-4}$ and we get the desired result. 
Theorem 5.4 Let $f: X \rightarrow Y$ be a mapping satisfying (5.1) and $f(0)=0$ for which there exists a function $\varphi: X^{2} \rightarrow[0, \infty)$ such that

$$
\varphi(x, y) \leq \frac{\alpha}{16} \varphi(2 x, 2 y)
$$

for all $x, y \in X$ with $x \perp y$. If, for each $x \in X$, the mapping $f(t x)$ is continuous in $t \in \mathbb{R}$, then there exists a unique orthogonally A-quartic mapping $P: X \rightarrow Y$ such that

$$
\|f(x)-P(x)\|_{Y} \leq \frac{\alpha}{32-32 \alpha} \varphi(x, 0)
$$

for all $x \in X$.

Proof Let $(S, d)$ be the generalized metric space defined in the proof of Theorem 2.2.

Now, we consider the linear mapping $J: S \rightarrow S$ such that

$$
J g(x):=16 g\left(\frac{x}{2}\right)
$$

for all $x \in X$. It follows from (5.2) that $d(f, J f) \leq \frac{\alpha}{32}$. So, we obtain inequality (5.4). The rest of the proof is similar to the proofs of Theorems 2.2 and 3.2.

Corollary 5.5 Let $\theta$ be a positive real number and $p$ be a real number with $p>4$. Let $f: X \rightarrow Y$ be a mapping satisfying (5.3). If, for each $x \in X$, the mapping $f(t x)$ is continuous in $t \in \mathbb{R}$, then there exists a unique orthogonally A-quartic mapping $P: X \rightarrow Y$ such that

$$
\|f(x)-P(x)\|_{Y} \leq \frac{\theta}{2\left(2^{p}-16\right)}\|x\|^{p}
$$

for all $x \in X$.

Proof The proof follows from Theorem 5.4 by taking $\varphi(x, y)=\theta\left(\|x\|^{p}+\|y\|^{p}\right)$ for all $x, y \in X$ with $x \perp y$. Then we can choose $\alpha=2^{4-p}$ and we get the desired result.

\section{Conclusions}

Using a fixed point method, we have proved the Hyers-Ulam stability of the orthogonally Jensen additive functional equation, of the orthogonally Jensen quadratic functional equation, of the orthogonally cubic functional equation and of the orthogonally quartic functional equation in orthogonality modules over a unital Banach algebra.

Competing interests

The authors declare that they have no competing interests.

Authors' contributions

All authors conceived of the study, participated in its design and coordination, drafted the manuscript, participated in the sequence alignment, and read and approved the final manuscript.

\section{Author details}

'Department of Mathematics, Research Institute for Natural Sciences, Hanyang University, Seoul, 133-791, Korea.

${ }^{2}$ Department of Mathematics Education and the RINS, Gyeongsang National University, Chinju, 660-701, Korea.

${ }^{3}$ Department of Mathematics, Daejin University, Pocheon, Kyeonggi 487-711, Korea. 


\section{References}

1. Pinsker, AG: Sur une fonctionnelle dans l'espace de Hilbert. C. R. (Dokl.) Acad. Sci. URSS 20, 411-414 (1938)

2. Sundaresan, K: Orthogonality and nonlinear functionals on Banach spaces. Proc. Am. Math. Soc. 34, 187-190 (1972)

3. Gudder, S, Strawther, D: Orthogonally additive and orthogonally increasing functions on vector spaces. Pac. J. Math. $58,427-436(1975)$

4. Rätz, J: On orthogonally additive mappings. Aequ. Math. 28, 35-49 (1985)

5. Rätz, J, Szabó, G: On orthogonally additive mappings, IV. Aequ. Math. 38, 73-85 (1989)

6. Alonso, J, Benítez, C: Orthogonality in normed linear spaces: a survey, I. Main properties. Extr. Math. 3, 1-15 (1988)

7. Alonso, J, Benítez, C: Orthogonality in normed linear spaces: a survey, II. Relations between main orthogonalities. Extr. Math. 4, 121-131 (1989)

8. Birkhoff, G: Orthogonality in linear metric spaces. Duke Math. J. 1, 169-172 (1935)

9. Carlsson, SO: Orthogonality in normed linear spaces. Ark. Mat. 4, 297-318 (1962)

10. Cho, Y, Diminnie, CR, Freese, RW, Andalafte, EZ: Isosceles orthogonal triples in linear 2-normed spaces. Math. Nachr. 157, 225-234 (1992)

11. Diminnie, CR: A new orthogonality relation for normed linear spaces. Math. Nachr. 114, 197-203 (1983)

12. James, RC: Orthogonality and linear functionals in normed linear spaces. Trans. Am. Math. Soc. 61, $265-292$ (1947)

13. Ulam, SM: Problems in Modern Mathematics. Wiley, New York (1960)

14. Hyers, DH: On the stability of the linear functional equation. Proc. Natl. Acad. Sci. USA 27, $222-224$ (1941)

15. Rassias, TM: On the stability of the linear mapping in Banach spaces. Proc. Am. Math. Soc. 72, 297-300 (1978)

16. Czerwik, S: Functional Equations and Inequalities in Several Variables. World Scientific, Singapore (2002)

17. Czerwik, S: Stability of Functional Equations of Ulam-Hyers-Rassias Type. Hadronic Press, Palm Harbor (2003)

18. Hyers, DH, Isac, G, Rassias, TM: Stability of Functional Equations in Several Variables. Birkhäuser, Basel (1998)

19. Jung, S: Hyers-Ulam-Rassias Stability of Functional Equations in Mathematical Analysis. Hadronic Press, Palm Harbor (2001)

20. Rassias, TM: Functional Equations, Inequalities and Applications. Kluwer Academic, Dordrecht (2003)

21. Ger, R, Sikorska, J: Stability of the orthogonal additivity. Bull. Pol. Acad. Sci., Math. 43, 143-151 (1995)

22. Skof, F: Proprietà locali e approssimazione di operatori. Rend. Semin. Mat. Fis. Milano 53, 113-129 (1983)

23. Cholewa, PW: Remarks on the stability of functional equations. Aequ. Math. 27, 76-86 (1984)

24. Czerwik, S: On the stability of the quadratic mapping in normed spaces. Abh. Math. Semin. Univ. Hamb. 62, 59-64 (1992)

25. Cho, Y, Park, C, Rassias, TM, Saadati, R: Inner product spaces and functional equations. J. Comput. Anal. Appl. 13 296-304 (2011)

26. Cho, Y, Saadati, R: Lattice non-Archimedean random stability of ACQ functional equation. Adv. Differ. Equ. 2011, Art. ID 31 (2011)

27. Najati, A, Kang, J, Cho, Y: Local stability of the Pexiderized Cauchy and Jensen's equations in fuzzy spaces. J. Inequal. Appl. 2011, Art. ID 78 (2011)

28. Rassias, TM: On the stability of the quadratic functional equation and its applications. Stud. Univ. Babeş-Bolyai, Math 43, 89-124 (1998)

29. Rassias, TM: The problem of S.M. Ulam for approximately multiplicative mappings. J. Math. Anal. Appl. 246, 352-378 (2000)

30. Rassias, TM: On the stability of functional equations in Banach spaces. J. Math. Anal. Appl. 251, 264-284 (2000)

31. Saadati, R, Cho, Y, Vahidi, J: The stability of the quartic functional equation in various spaces. Comput. Math. Appl. 60, 1994-2002 (2010)

32. Vajzović, F: Über das Funktional $H$ mit der Eigenschaft: $(x, y)=0 \Rightarrow H(x+y)+H(x-y)=2 H(x)+2 H(y)$. Glas. Mat. 2(22) 73-81 (1967)

33. Drljević, F: On a functional which is quadratic on A-orthogonal vectors. Publ. Inst. Math. (Belgr.) 54, 63-71 (1986)

34. Fochi, M: Functional equations in A-orthogonal vectors. Aequ. Math. 38, 28-40 (1989)

35. Moslehian, MS: On the orthogonal stability of the Pexiderized quadratic equation. J. Differ. Equ. Appl. 11, 999-1004 (2005)

36. Moslehian, MS: On the stability of the orthogonal Pexiderized Cauchy equation. J. Math. Anal. Appl. 318, 211-223 (2006)

37. Szabó, G: Sesquilinear-orthogonally quadratic mappings. Aequ. Math. 40, 190-200 (1990)

38. Moslehian, MS, Rassias, TM: Orthogonal stability of additive type equations. Aequ. Math. 73, 249-259 (2007)

39. Park, C, Cho, Y, Kenary, HA: Orthogonal stability of a generalized quadratic functional equation in non-Archimedean spaces. J. Comput. Anal. Appl. 14, 526-535 (2012)

40. Park, C: Orthogonal stability of an additive-quadratic functional equation. Fixed Point Theory Appl. 2011, Art. ID 66 (2011)

41. Jun, K, Kim, H: The generalized Hyers-Ulam-Rassias stability of a cubic functional equation. J. Math. Anal. Appl. 274 867-878 (2002)

42. Lee, S, Im, S, Hwang, I: Quartic functional equations. J. Math. Anal. Appl. 307, 387-394 (2005)

43. Diaz, J, Margolis, B: A fixed point theorem of the alternative for contractions on a generalized complete metric space. Bull. Am. Math. Soc. 74, 305-309 (1968)

44. Cădariu, L, Radu, V: Fixed points and the stability of Jensen's functional equation. J. Inequal. Pure Appl. Math. 4(1), Art. ID 4 (2003)

45. Isac, G, Rassias, TM: Stability of $\psi$-additive mappings: applications to nonlinear analysis. Int. J. Math. Math. Sci. 19 , 219-228 (1996)

46. Najati, A, Cho, Y: Generalized Hyers-Ulam stability of the pexiderized Cauchy functional equation in non-Archimedean spaces. Fixed Point Theory Appl. 2011, Art. ID 309026 (2011)

47. Park, C: Fixed points and Hyers-Ulam-Rassias stability of Cauchy-Jensen functional equations in Banach algebras. Fixed Point Theory Appl. 2007, Art. ID 50175 (2007)

48. Park, C: Generalized Hyers-Ulam-Rassias stability of quadratic functional equations: a fixed point approach. Fixed Point Theory Appl. 2008, Art. ID 493751 (2008) 
49. Radu, V: The fixed point alternative and the stability of functional equations. Fixed Point Theory 4, 91-96 (2003)

50. Miheț, D, Radu, V: On the stability of the additive Cauchy functional equation in random normed spaces. J. Math. Anal. Appl. 343, 567-572 (2008)

51. Cădariu, L, Radu, V: On the stability of the Cauchy functional equation: a fixed point approach. Grazer Math. Ber. 346 43-52 (2004)

52. Cădariu, L, Radu, V: Fixed point methods for the generalized stability of functional equations in a single variable. Fixed Point Theory Appl. 2008, Art. ID 749392 (2008)

53. Cho, Y, Kang, J, Saadati, R: Fixed points and stability of additive functional equations on the Banach algebras. J. Comput. Anal. Appl. 14, 1103-1111 (2012)

54. Jung, Y, Chang, I: The stability of a cubic type functional equation with the fixed point alternative. J. Math. Anal. Appl. 306, 752-760 (2005)

55. Mirzavaziri, M, Moslehian, MS: A fixed point approach to stability of a quadratic equation. Bull. Braz. Math. Soc. 37 , $361-376(2006)$

doi:10.1186/1687-1847-2012-173

Cite this article as: Park et al.: Orthogonal stability of functional equations with the fixed point alternative. Advances in Difference Equations 2012 2012:173.

Submit your manuscript to a SpringerOpen ${ }^{\circ}$ journal and benefit from:

- Convenient online submission

- Rigorous peer review

Immediate publication on acceptance

- Open access: articles freely available online

- High visibility within the field

- Retaining the copyright to your article

Submit your next manuscript at $>$ springeropen.com 